\title{
A WELL-CONDITIONED BOUNDARY INTEGRAL EQUATION FOR TRANSMISSION PROBLEMS OF ELECTROMAGNETISM
}

\author{
DAVID LEVADOUX, FLORENCE MILLOT AND SÉBASTIEN PERNET \\ Communicated by Ivan Graham
}

\begin{abstract}
We propose a new well-conditioned boundary integral equation to solve transmission problems of electromagnetism. This equation is well posed and appears as a compact perturbation of the identity leading to fast iterative solutions without the help of any preconditioner. Some numerical experiments confirm this result.
\end{abstract}

1. Introduction. Boundary integral methods (BIM) are commonly used for solving scattering problems of arbitrarily shaped three dimensional obstacles and also for antenna design. Their popularity is due to a combination of many factors. Firstly, the solutions of BIM fulfill causality and radiation conditions automatically. Secondly, it is only necessary to discretize the boundaries of the computational domain, and the simulation requires a smaller number of unknowns than finite element methods or finite difference methods. One of the main drawbacks of using a BIM is that, after discretization, it results in a dense system of linear equations. Moreover, if the size of the obstacle increases, the solutions can only be obtained by means of some iterative methods coupled with the multilevel fast multipole method (FMM). The convergence of the iterative methods is directly linked to the choice of the integral formulation. So, the main difficulty is to choose the best boundary equation in the sense that this equation gives rise to a wellconditioned linear system and also in the sense that the solutions must be accurate. An algebraic preconditioner [11] is generally used to im-

2010 AMS Mathematics subject classification. Primary 31B10, 65F08, 76M15, $78 \mathrm{M} 16$.

Keywords and phrases. Integral equation, boundary element method, transmission problem, preconditioner, GMRES, fast multipole method.

Received by the editors on November 28, 2014, and in revised form on May 29, 2015.

DOI: $10.1216 /$ JIE-2015-27-3-431

Copyright (C)2015 Rocky Mountain Mathematics Consortium 
prove the convergence of the iterative solver. Unfortunately, this kind of approach loses its effectiveness when the frequency increases or the meshes become denser with respect to the wavelength.

A few years ago, a new practice of BIM emerged which consists of building non standard integral equations that deliver well-conditioned linear systems after discretization and are able to be efficiently solved with no preconditioner.

At the origin of this approach was a generalization of the BrackhageWerner equation of acoustics in which the coupling coefficient between the single and double layer potentials was replaced by an approximation of the Dirichlet-to-Neumann operator $[\mathbf{1 7}, \mathbf{2 0}, \mathbf{2 1}]$. This coupling operator played the role of a preconditioner analytically regularizing the badly conditioned single layer potential. At the same time, this equation was shown to belong to a new class of integral equations, the so-called generalized combined source integral equations (GCSIE), opening the way to build some intrinsically well-conditioned equations for many other boundary conditions.

Actually, the GCSIE formalism depends on the choice of a regularizing operator $\widetilde{R}$ whose vocation is to approximate as closely as possible the boundary operator $R$ linking one component of the Cauchy data to the other. A crucial feature of this approach is that, if the approximation $\widetilde{R}$ is exact, the resulting equation becomes trivial. In other words, the GCSIE formalism allows finding the optimal preconditioner whose $\widetilde{R}$ must be a good approximation.

A powerful technique for finding such an approximation consists of building an operator $\widetilde{R}$ with the same principal symbol as $R$. But this problem is very ill-posed, one reason being that the principal symbol of an operator is unchanged under compact perturbation. Therefore, a second constraint of a more physical nature is introduced demanding $\widetilde{R}$ to coincide with $R$ on flat parts of the boundary.

When this program is realized, the first requirement on the symbolic structure of $\widetilde{R}$ leads to an equation being a compact perturbation $K$ of identity, and the second requirement, to a $K$ with a small operator norm.

Therefore, this type of equation is well adapted to an iterative solution and, if the spectral behavior of the equation is well restored 
to the discrete level, then the convergence rate is independent from the space refinement $[\mathbf{9}, \mathbf{1 0}]$. That is why the GCSIE equations are attractive, compared to the classical algebraic preconditioners like SPAI (SParse approximate inverse) where parameters depending on the frequency and the quality of the mesh are not obviously taken into account and need to be tuned very carefully. Indeed, The GCSIE simplifies the work of the "end user" by regularizing the pathological behavior of operations at the continuous level.

Several constructions of $\widetilde{R}$ operators have been successfully tested in acoustics or electromagnetism for Dirichlet or Neumann problems $[\mathbf{1}, \mathbf{2}, \mathbf{3}, \mathbf{6}, \mathbf{7}, \mathbf{1 4}, \mathbf{1 5}]$, and more recently Robin problems $[\mathbf{2 2}, \mathbf{2 5}]$. The numerical results are impressive in terms of reducing the number of iterations and the CPU time. The GCSIE formalism can also be extended to transmission problems leading in acoustics to well-posed and well-conditioned equations $[4,5]$. In electromagnetism, a first integral equation has already been proposed [18] but with no proof of its theoretical well posedness nor its numerical efficiency. The work we present here fills this gap, thanks to the use of a simpler $\widetilde{R}$ operator.

The paper is divided as follows. In Section 2, we present the transmission problem to be solved, and we recall some background on integral equations in electromagnetism. In Section 3, we propose and theoretically analyze a GCSIE formulation for the transmission problem. In particular, we prove that our choice leads to an equation well-posed at any frequency and whose the underlying operator is a compact perturbation of the identity. The final Section 4 is devoted to numerical results. A comparison with the so-called PMCHWT (Poggio-Miller-Chan-Harrington-Wu-Tsai) integral equation shows the performance of the new GCSIE formulation.

\section{Transmission problem and background on integral equa-} tions. In this section, we define at first the transmission problem considered in this paper, and then we give some well-known results about the integral formalism in electromagnetism. In particular, we recall the so-called PMCHWT (Poggio-Miller-Chan-Harrington-Wu-Tsai) integral equation.

2.1. Transmission problem. Let $\Omega_{1}$ and $\Omega_{2}:=\mathbb{R}^{3} \backslash \overline{\Omega_{1}}$ be two open domains of $\mathbb{R}^{3}$ containing homogeneous dielectric materials with 
electric permittivity and magnetic permeability $\left(\varepsilon_{j} ; \mu_{j}\right), j=1,2$. We assume that $\Omega_{2}$ is bounded. The outward pointing normal vector on the boundary $\Gamma$ of $\Omega_{j}$ is $\mathbf{n}_{j}$ and $\mathbf{n}:=\mathbf{n}_{2}$. The impedance and the wave number of each medium are, respectively, given by $z_{j}:=\sqrt{\mu_{j} / \varepsilon_{j}}$ and $k_{j}:=\omega \sqrt{\mu_{j} \varepsilon_{j}}$, where $\omega$ is the frequency. Finally, if $\mathbf{U}: \mathbb{R}^{3} \rightarrow \mathbb{R}^{3}$ is a vector field defined on $\Omega_{1} \cup \Omega_{2}$, we adopt the notation $\gamma_{j} \mathbf{U}:=\mathbf{n} \times \mathbf{U}_{j \mid \Gamma}$, where $\mathbf{U}_{j}:=\mathbf{U}_{\mid \Omega_{j}}$. The symbol $\oplus$ is used to stick together two fields separately defined on $\Omega_{1}$ and $\Omega_{2}$, e.g., $\mathbf{U}=\mathbf{U}_{1} \oplus \mathbf{U}_{2}$.

For the sequel, we will say that a vector field $(\mathbf{E}, \mathbf{H})$ on $\mathbb{R}^{3} \backslash \Gamma$ is a radiating electromagnetic field if it satisfies the Maxwell equations

$$
\begin{aligned}
\nabla \times \mathbf{E}_{j}-i k_{j} z_{j} \mathbf{H}_{j}=0 & \text { in } j(j=1,2) \\
\nabla \times \mathbf{H}_{j}+i k_{j} z_{j}^{-1} \mathbf{E}_{j}=0 & \text { in } j(j=1,2)
\end{aligned}
$$

and the radiation condition

$$
\lim \left(\mathbf{E}_{1}(x)+z_{1} \frac{x}{|x|} \times \mathbf{H}_{1}(x)\right)=0 \quad \text { when }|x| \rightarrow+\infty .
$$

Given a couple $\left(\mathbf{u}_{0}, \mathbf{v}_{0}\right)$ of tangential vector fields on $\Gamma$ we search a radiating electromagnetic field $(\mathbf{E}, \mathbf{H})$ satisfying in addition the transmission condition $\gamma_{1}\left(\mathbf{E}_{1}, \mathbf{H}_{1}\right)-\gamma_{2}\left(\mathbf{E} 2, \mathbf{H}_{2}\right)=\left(\mathbf{u}_{0}, \mathbf{v}_{0}\right)$ read as

$$
\tau(\mathbf{E}, \mathbf{H})=\left(\mathbf{u}_{0}, \mathbf{v}_{0}\right)
$$

with the notation $\tau:=\gamma_{1}-\gamma_{2}$. When $\left(\mathbf{E}^{\text {inc }}, \mathbf{H}^{\text {inc }}\right)$ is a plane wave impinging $\Omega_{2}$ from $\Omega_{1}$ classic conditions are:

$$
\gamma_{1}\left(\mathbf{E}_{1}, \mathbf{H}_{1}\right)-\gamma_{2}\left(\mathbf{E}_{2}, \mathbf{H}_{2}\right)=-\gamma_{1}\left(\mathbf{E}^{\text {inc }}, \mathbf{H}^{\text {inc }}\right),
$$

leading to a well-posed transmission problem $[\mathbf{8}, \mathbf{2 4}]$.

2.2. Potentials and boundary operators. Classical potentials in $\mathbb{R}^{3} \backslash \Gamma$ are given by:

$$
\mathcal{T}=\frac{1}{i k} \nabla \times \nabla \times \mathcal{G} \quad \text { and } \quad \mathcal{K}=\nabla \times \mathcal{G},
$$

where $\mathcal{G}$ stands for the vector potential, depending on a given wavenumber $k$, which to a tangent vector-field $\mathbf{u}$ on $\Gamma$ associates the vector-field defined on $\mathbb{R}^{3} \backslash \Gamma$ by

$$
\mathcal{G} \mathbf{u}(x)=-\frac{1}{4 \pi} \int_{\Gamma} \frac{e^{i k|x-y|}}{|x-y|} \mathbf{u}(y) d y .
$$


The traces of $\mathcal{T}$ and $\mathcal{K}$ potentials yield two fundamental boundary operators

$$
\mathcal{T}:=-\mathbf{n} \times \gamma_{j} \mathcal{T}, \quad K:=-\mathbf{n} \times\left(\gamma_{j} \mathcal{K}+\mathrm{Id} / 2\right),
$$

which are pseudo-differential operators of orders, respectively, 1 and -1 when the boundary $\Gamma$ is smooth [26]. These definitions do not depend on the domain $\Omega_{j}$ from which are taken the traces, reflecting a specific behavior of the tangential components of $\mathcal{T}$ and $\mathcal{K}$ across the boundary: no jump for $\mathcal{T}$ to the contrary of $\mathcal{K}$ whose jump is precisely $K$. On the other hand, a well known and useful description for $\mathcal{T}$ is

$$
T=\frac{1}{i k}\left(\nabla G^{s} \nabla \cdot+k^{2} G^{v}\right)
$$

where $G^{v}$ (respectively, $G^{s}$ ) is the tangential (respectively, Dirichlet) trace of $\mathcal{G}$.

Both $G^{s}$ and $G^{v}$ will be simply referred to as $G$ if the context is clear, and $\mathbf{n} \times T$ and $\mathbf{n} \times K$ will be noted as, respectively, $T^{\times}$and $K^{\times}$. Furthermore, when we will have to specify the wavenumber $k_{j}$ to which are related these operators, we will append the subscript $j$ to their symbolic letter (e.g., $T_{j}, K_{j}, G_{j}$, etc.). Such operators with no subscript will mean that the expression they compose is indifferently true either with $j=1$ or 2 .

If $(\mathbf{E}, \mathbf{H})$ is a radiating electromagnetic field with $k_{1}=k_{2}=: k$, then we have the following Stratton-Chu formulas [12]:

$$
\mathbf{C}_{j} \gamma_{j}(\mathbf{E}, \mathbf{H})= \begin{cases}(\mathbf{E}, \mathbf{H}) & \text { on } \Omega_{j} \\ 0 & \text { on } \Omega_{i}(i \neq j),\end{cases}
$$

where

$$
\mathbf{C}_{1}:=\left(\begin{array}{cc}
-\mathcal{K} & z \mathcal{T} \\
-\frac{1}{z} \mathcal{T} & -\mathcal{K}
\end{array}\right), \quad \mathbf{C}_{2}:=-\mathbf{C}_{1} .
$$

Exterior and interior tangential traces of theses potentials lead to the boundary operators

$$
C j:=j C j
$$

named as Calderón projectors and verifying

$$
C_{1} C_{2}=0 .
$$


From definitions (7) and (11) comes an expression of $C_{j}$ in function of the basic operators $T$ and $K$ :

$$
\begin{aligned}
C_{1} & =\left(\begin{array}{cc}
\frac{\mathrm{Id}}{2}-K^{\times} & z T^{\times} \\
-\frac{1}{z} T^{\times} & \frac{\mathrm{Id}}{2}-K^{\times}
\end{array}\right) \\
C_{2} & =\left(\begin{array}{cc}
\frac{\mathrm{Id}}{2}+K^{\times} & -z T^{\times} \\
\frac{1}{z} T^{\times} & \frac{\mathrm{Id}}{2}+K^{\times}
\end{array}\right)
\end{aligned}
$$

Expanding $C_{j}$ in (12) with (13) gives the so-called Calderón relations

$$
\begin{aligned}
\left(T^{\times}\right)^{2} & =\frac{\mathrm{Id}}{4}+\left(K^{\times}\right)^{2} \\
T^{\times} K^{\times} & =-K^{\times} T^{\times} .
\end{aligned}
$$

From now on in the sequel $\mathbf{C}_{1}$ and $\mathbf{C}_{2}$ are built, respectively, with the given wave numbers $k_{1}$ and $k_{2}$ of the Maxwell equations (1) governing the transmission problem we want to solve. Idem for the projectors $C_{1}$ and $C_{2}$.

2.3. A classic integral equation. The derivation of an integral equation to solve (1)-(4) is based on the Stratton-Chu formulas. Applying (9) to the solution $\left(\mathbf{E}_{j}, \mathbf{H}_{j}\right)$ of (1)-(4) and to the incident plane wave $\left(\mathbf{E}^{\text {inc }}, \mathbf{H}^{\text {inc }}\right)$ gives, respectively,

$$
\begin{aligned}
\gamma_{1} \mathbf{C}_{1} \gamma_{1}\left(\mathbf{E}_{1}, \mathbf{H}_{1}\right)-\gamma_{2} \mathbf{C}_{2} \gamma_{2}\left(\mathbf{E}_{2}, \mathbf{H}_{2}\right) & =-\gamma_{1}\left(\mathbf{E}^{\text {inc }}, \mathbf{H}^{\text {inc }}\right) \\
\gamma_{1} \mathbf{C}_{1} \gamma_{1}\left(\mathbf{E}^{\text {inc }}, \mathbf{H}^{\text {inc }}\right) & =0 .
\end{aligned}
$$

Converting exterior traces to interior ones in (16) with (4) and adding (17) to (16) one obtains the PMCHWT integral equation [16]

$$
\left(C_{1}-C_{2}\right)(\mathbf{M}, \mathbf{J})=-\gamma_{1}\left(\mathbf{E}^{\text {inc }}, \mathbf{H}^{\text {inc }}\right)
$$

whose solutions $(\mathbf{M}, \mathbf{J})=\left(\gamma_{2} \mathbf{E}, \gamma_{2} \mathbf{H}\right)$ are the Cauchy data of the electromagnetic field in the dielectric $\Omega_{2}$.

\section{Intrinsically well-conditioned integral equations.}

3.1. Principle of the construction and objectives. The starting point for building regularized boundary integral equations with the GCSIE formalism is based on some obvious but crucial remarks. Since 
the transmission problem (1)-(3) is well posed there exists a potential $\mathcal{R}$ such that $\mathcal{R}\left(\mathbf{u}_{0}, \mathbf{v}_{0}\right)=(\mathbf{E}, \mathbf{H})$. Therefore, by construction of $\mathcal{R}$,

$$
\tau \mathcal{R}=\mathrm{Id}
$$

Taking traces of the electromagnetic field radiated by $\mathcal{R}$ in each domain $\Omega_{j}$ leads to a $2 \times 2$ matrix of boundary operators:

$$
R_{j}:=\gamma_{j} \mathcal{R}
$$

We denote by $R:=\left(R_{1}, R_{2}\right)$. The relation (19) giving $R_{1}-R_{2}=\mathrm{Id}$,

$$
R=\left(R_{1}, R_{1}-\mathrm{Id}\right) .
$$

From the Stratton-Chu formulas (9) and the definition of $R_{j}(20)$ we have

$$
\begin{aligned}
(\mathbf{E}, \mathbf{H}) & =\mathbf{C}_{1} \gamma_{1}(\mathbf{E}, \mathbf{H}) \oplus \mathbf{C}_{2} \gamma_{2}(\mathbf{E}, \mathbf{H}) \\
& =\left(\mathbf{C}_{1} R_{1} \oplus \mathbf{C}_{2} R_{2}\right)\left(\mathbf{u}_{0}, \mathbf{v}_{0}\right) .
\end{aligned}
$$

Finally, the transmission condition $\tau(\mathbf{E}, \mathbf{H})=\left(\mathbf{u}_{0}, \mathbf{v}_{0}\right)$ gives the crucial relation

$$
\tau\left(\mathbf{C}_{1} R_{1} \oplus \mathbf{C}_{2} R_{2}\right)=\text { Id }
$$

Now, let $\widetilde{R}=\left(\widetilde{R}_{1}, \widetilde{R}_{2}\right)$ be an approximation of $R$. We decide to look for the solution $(\mathbf{E}, \mathbf{H})$ of the transmission problem (1)-(3) under the form

$$
(\mathbf{E}, \mathbf{H})=\left(\mathbf{C}_{1} \widetilde{R}_{1} \oplus \mathbf{C}_{2} \widetilde{R}_{2}\right)(\mathbf{u}, \mathbf{v})
$$

where $(\mathbf{u}, \mathbf{v})$ are a couple of current distributions on $\Gamma$ acting as a source excitation of the potential $\mathbf{C}_{1} \widetilde{R}_{1} \oplus \mathbf{C}_{2} \widetilde{R}_{2}$. Therefore, in order to find a source $(\mathbf{u}, \mathbf{v})$ radiating the field solution of (1)-(3), we have to solve the resulting source integral equation

$$
\tau\left(\mathbf{C}_{1} \widetilde{R}_{1} \oplus \mathbf{C}_{2} \widetilde{R}_{2}\right)(\mathbf{u}, \mathbf{v})=\left(\mathbf{u}_{0}, \mathbf{v}_{0}\right) .
$$

Because of the crucial relation $(22)$, if $\widetilde{R}=R$, the new equation (24) becomes trivial. Therefore, we take up the challenge that if we succeed in building an operator $\widetilde{R}$ sufficiently "close" to $R$, the resulting equation will be a "small" perturbation of the identity produced after discretization of a well-conditioned linear system. 
3.2. Convenient form of the regularizing operator $R$. We recall that there exists a boundary operator $Y_{j}(j=1,2)$, called admittance, such that, for all radiating electromagnetic fields $\left(\mathbf{E}_{j}, \mathbf{H}_{j}\right)$,

$$
Y_{j}\left(\gamma_{j} \mathbf{E}_{j}\right)=\gamma_{j} \mathbf{H}_{j}
$$

Actually, admittance links the first Cauchy data of $\mathbf{E}_{j}$ given by $\gamma_{j} \mathbf{E}_{j}$ to the second one being $\gamma_{j} \mathbf{H}_{j}$. As $\mathbf{E}$ and $\mathbf{H}$ play symmetrical roles in (1), one can easily show that

$$
Y_{j}^{2}=-\mathrm{Id} / z_{j}^{2}
$$

Proposition 3.1. The $2 \times 2$ matrix $R_{1}$ can be read as

$$
R_{1}=\left(\begin{array}{cc}
A & -z_{1}^{2} Y_{1} B \\
Y_{1} A & B
\end{array}\right),
$$

where $A:=\left(\operatorname{Id}+z_{2}^{2} Y_{2} Y_{1}\right)^{-1}$ and $B:=\left(\operatorname{Id}+z_{1}^{2} Y_{2} Y_{1}\right)^{-1}$.

Proof. If $R_{k l}^{j}$ denotes the operator-coefficient $k l$ of $R_{j},(19)$ is equivalent to

$$
\begin{aligned}
R_{11}^{1}-R_{11}^{2} & =\mathrm{Id} & R_{12}^{1} & =R_{12}^{2} \\
R_{21}^{1} & =R_{21}^{2} & R_{22}^{1}-R_{22}^{2} & =\mathrm{Id} .
\end{aligned}
$$

As the two rows of $R_{j}$ are the Cauchy data of the field radiated by $R$ which are linked to each other following (25), we have

$$
Y_{j} R_{11}^{j}=R_{21}^{j} \quad Y_{j} R_{12}^{j}=R_{22}^{j} .
$$

Therefore, $R_{21}^{1}=Y_{1} A$ and $R_{12}^{1}=Y_{1}^{-1} B=-z_{1}^{2} Y_{1} B$ with the help of (26).

Multiplying $R_{11}^{1}-R_{11}^{2}=$ Id by $Y_{2}$ and, using (29), gives $Y_{2} R_{11}^{1}-$ $R_{21}^{2}=Y_{2}$. Changing $R_{21}^{2}$ in $R_{21}^{1}$ and reusing $(29)$ leads to $\left(Y_{2}-Y_{1}\right) R_{11}^{1}=$ $Y_{2}$. Finally, $A=R_{11}=\left(Y_{2}-Y_{1}\right)^{-1} Y_{2}=\left(\mathrm{Id}-Y_{2}^{-1} Y_{1}\right)^{-1}=$ $\left(\mathrm{Id}+z_{2}^{2} Y_{2} Y_{1}\right)^{-1}$ thanks to (26). $B$ is found in a similar way.

We now have to equip the GCSIE (24) with an $\widetilde{R}$ operator being a relevant approximation of $R$. Due to (21), it is quite natural to search $\widetilde{R}$ under the form $\left(\widetilde{R}_{1}, \widetilde{R}_{1}-\mathrm{Id}\right)$ where $\widetilde{R}_{1}$ is defined by the expression (27) in which $A, B$ and $Y_{1}$ are replaced by some counterpart approximations. 
The two next paragraphs are devoted to finding such approximations with the help of the pseudo-differential calculus. Therefore, in order to use this tool, the surface $\Gamma$ is supposed smooth. $\Psi^{s}$ denotes the class of classical pseudodifferential operators of order $s$ on $\Gamma$.

\subsection{Approximations of $A$ and $B$.}

Proposition 3.2. The admittance operators $Y_{1}$ and $Y_{2}$ are of order 1 and

$$
\begin{aligned}
& z_{1} Y_{1}=-2 T_{1}^{\times}+4 K_{1}^{\times} T_{1}^{\times} \bmod \Psi^{-1} \\
& z_{2} Y_{2}=2 T_{2}^{\times}+4 K_{2}^{\times} T_{2}^{\times} \quad \bmod \Psi^{-1}
\end{aligned}
$$

Proof. As for $R_{j}$, a similar relation to (29) stands for $C_{j}$ (the reason is the same). Therefore, (13) leads to

$$
\begin{aligned}
& z_{1} Y_{1} T_{1}^{\times}=\frac{\mathrm{Id}}{2}-K_{1}^{\times} \\
& z_{2} Y_{2} T_{2}^{\times}=-\frac{\mathrm{Id}}{2}-K_{2}^{\times} .
\end{aligned}
$$

Using (32) and the Calderón formula (14), we get

$$
-z_{1} \frac{1}{4} Y_{1}=\frac{1}{2} T_{1}^{\times}-K_{1}^{\times} T_{1}^{\times}-z_{1} Y_{1}\left(K_{1}^{\times}\right)^{2} .
$$

The orders of $K_{1}^{\times}$and $\left(K_{1}^{\times}\right)^{2}$ being, respectively, -1 and $-2,-(1 / 4) Y_{1}$ and $(1 / 2) T_{1}^{\times}$have the same principal symbol and, therefore, the order of $Y_{1}$ is 1 . As $Y_{1}\left(K_{1}^{\times}\right)^{2}$ is of order -1 , one has (30). The proof of (31) is the same.

The well-known Helmholtz decomposition states that the space $H_{T}^{0}$ of $L^{2}$ tangential fields on $\Gamma$ splits into a direct sum of two closed orthogonal subspaces:

$$
H_{T}^{0}=\mathbf{n} \times \nabla H^{1} \oplus^{\perp} \nabla H^{1}
$$

where $H^{1}$ is the usual Sobolev space of order 1 on $\Gamma$. Moreover, it is possible to give to the underlying projectors of this decomposition a more explicit form with the help of the Moore-Penrose pseudoinverse of Laplace-Beltrami operator $\Delta$. Indeed, if $H_{\bullet}^{s}(s>0)$ denotes the space of fields $u \in H^{s}$ with $\int u d \Gamma=0, \Delta$ induces a one-to-one mapping $\widetilde{\Delta}$ 
from $H_{\bullet}^{2}$ to $H_{\bullet}^{0}$. Taking $\Delta^{1}$ as the pseudoinverse $w \widetilde{\Delta}^{-1} \prod$, where $\prod$ is the orthogonal projector of $H^{0}$ onto $H_{\bullet}^{0}$, one has:

Proposition 3.3. Projectors $\left(\prod_{\mathrm{loop}}, \prod_{\mathrm{star}}\right)$ of the Helmholtz decomposition (33) read as

$$
\begin{aligned}
& \prod_{\text {loop }}=D_{\text {loop }} P_{\text {loop }} \\
& \prod_{\text {star }}=D_{\text {star }} P_{\text {star }}
\end{aligned}
$$

where

$$
\begin{aligned}
P_{\text {loop }} & =\Delta^{-1} \nabla \cdot \mathbf{n} \times, \quad P_{\text {star }}=\Delta^{-1} \nabla \\
D_{\text {loop }} & =-\mathbf{n} \times \nabla, \quad D_{\text {star }}=\nabla .
\end{aligned}
$$

Proof. We denote by $\left(\pi_{1}, \pi_{2}\right)$ the underlying projectors of the decomposition (33) by $\left(\pi_{1}, \pi_{2}\right)$. For all $\mathbf{u}=\mathbf{n} \times \nabla p$ with $p \in H^{1}(\Gamma)$, one has $\prod_{\text {loop }} \mathbf{u}=\mathbf{n} \times \nabla \Delta^{-1} \nabla \cdot \nabla p=\mathbf{n} \times \nabla\left(p-\int_{\Gamma} p(x) d x /|\Gamma|\right)=\mathbf{n} \times \nabla p=\mathbf{u}$. So $\prod_{\text {loop }} \pi_{1}=\pi_{1}$, and, as $\prod_{\text {loop }} \pi_{2}=0$, it is shown that $\prod_{\text {loop }}=$ $\prod_{\text {loop }}\left(\pi_{1}+\pi_{2}\right)=\pi_{1}$. A similar proof stands for $\prod_{\text {star }}=\pi_{2}$.

From now on, we will need to use the principal symbols of $G^{s}$ and $\Delta$ given as functions of a $\xi$ parameter being a cotangent vector on $\Gamma$ :

$$
\sigma_{G}(\xi)=-\|\xi\|^{-1} / 2, \quad \sigma_{\Delta}(\xi)=-\|\xi\|^{2} .
$$

These symbols, well known for a long time [26], are at the heart of the next result.

Proposition 3.4. The operator $Y_{2} Y_{1}$ is of order 0 and

$$
z_{1} z_{2} Y_{2} Y_{1}=\frac{k_{1}}{k_{2}} \prod_{\text {loop }}+\frac{k_{2}}{k_{1}} \prod_{\text {star }} \bmod \Psi^{-1}
$$

Proof. Firstly, let us verify that

$$
T_{2}^{\times} T_{1}^{\times}=-\frac{1}{4}\left(\frac{k_{1}}{k_{2}} \prod_{\text {loop }}+\frac{k_{2}}{k_{1}} \prod_{\text {star }}\right) \bmod \Psi^{-1} .
$$


Indeed, from (8), we have modulo $\Psi^{-1}$,

$$
\begin{aligned}
T_{2}^{\times} T_{1}^{\times} & =-\frac{1}{k_{1} k_{2}}\left(k_{1}^{2} \mathbf{n} \times \nabla G_{2} \nabla \cdot \mathbf{n} \times G_{1}+k_{2}^{2} \mathbf{n} \times G_{2} \mathbf{n} \times \nabla G_{1} \nabla \cdot\right) \\
& =-\frac{1}{k_{1} k_{2}}\left(k_{1}^{2} \mathbf{n} \times \nabla G_{2} G_{1} \nabla \cdot \mathbf{n} \times-k_{2}^{2} \nabla G_{2} G_{1} \nabla \cdot\right) .
\end{aligned}
$$

Then, equality (39) results from $-4 G_{1} G_{2}=\Delta^{-1} \bmod \Psi^{-3}$, because of (37), and from relations (34).

We denote by $S_{1}=z_{1} Y_{1}+2 T_{1}^{\times}$and $S_{2}=z_{2} Y_{2}-2 T_{2}^{\times}$, and compute

$$
\begin{aligned}
z_{1} z_{2} Y_{2} Y_{1} & =\left(S_{2}+2 T_{2}^{\times}\right)\left(S_{1}-2 T_{1}^{\times}\right) \\
& =S_{2} S_{1}-4 T_{2}^{\times} T_{1}^{\times}-2 S_{2} T_{1}^{\times}+2 T_{2}^{\times} S_{1} .
\end{aligned}
$$

From (30) and (31), one knows that, $\bmod ^{-1}, S_{1}=4 K_{1}^{\times} T_{1}^{\times}$and $S_{2}=4 K_{2}^{\times} T_{2}^{\times}$. Therefore, using the anti-commuting property (15) and the fact that $T_{1}^{\times} T_{2}^{\times}$is of order 0 as enlightened by (39), we can drop the -1 order operators $S_{1} S_{2}, S_{1} T_{2}^{\times}, T_{1}^{\times} S_{2}$, and again with the help of (39) one obtains (38).

Proposition 3.5. The operators $A$ and $B$ in (27) are of order 0 and are respectively equal modulo $\Psi^{-1}$ to

$$
\begin{aligned}
& \widetilde{A}:=\frac{z_{1} k_{2}}{z_{1} k_{2}+z_{2} k_{1}} \prod_{\text {loop }}+\frac{z_{1} k_{1}}{z_{1} k_{1}+z_{2} k_{2}} \prod_{\text {star }} \\
& \widetilde{B}:=\frac{z_{2} k_{2}}{z_{1} k_{1}+z_{2} k_{2}} \prod_{\text {loop }}+\frac{z_{2} k_{1}}{z_{1} k_{2}+z_{2} k_{1}} \prod_{\text {star }} .
\end{aligned}
$$

Proof. Using (38) and the relation $\mathrm{Id}=\prod_{\text {loop }}+\prod_{\text {star }}$ gives

$$
\begin{aligned}
\operatorname{Id}+z_{2}^{2} Y_{2} Y_{1}= & \frac{k_{1} z_{2}+k_{2} z_{1}}{k_{2} z_{1}} \prod_{\text {loop }} \\
& +\frac{k_{2} z_{2}+k_{1} z_{1}}{k_{1} z_{1}} \prod_{\text {star }} \bmod \Psi^{-1} .
\end{aligned}
$$

Therefore, $\left(\mathrm{Id}+z_{2}^{2} Y_{2} Y_{1}\right) \widetilde{A}=\mathrm{Id}+R$ with $R \in \Psi^{-1}$ and, because $A\left(\operatorname{Id}+z_{2}^{2} Y_{2} Y_{1}\right) \widetilde{A}=\widetilde{A}$, one has $A+A R=\widetilde{A}$, leading to (40). It is the same thing for (41). 
We leave it to the reader to verify a simple but very crucial relation for the sequel:

$$
\widetilde{A} \mathbf{n} \times+\mathbf{n} \times \widetilde{B}=\mathbf{n} \times .
$$

3.4. Approximation of $Y_{1}$. The Helmholtz decomposition allows us to handle any vectorial boundary operator $M$ as a $2 \times 2$ matrix of scalar boundary operators $M_{i j}$ as follows:

$$
M=\left(D_{\text {loop }} D_{\text {star }}\left(\begin{array}{ll}
M_{11} & M_{12} \\
M_{21} & M_{22}
\end{array}\right)\left(P_{\text {loop }} P_{\text {star }}\right) .\right.
$$

A meticulous pseudo-differential calculus, out of the scope of this paper, gives the following matrix representation of $G^{v}$ in the Helmholtz potentials:

$$
G^{v}=\left(\begin{array}{cc}
G^{s} & 0 \\
0 & G^{s}
\end{array}\right) \bmod \Psi^{-2}
$$

allowing us to easily state

$$
T^{\times}=\frac{1}{i k}\left(\begin{array}{cc}
0 & -G\left(\Delta+k^{2} \mathrm{Id}\right) \\
k^{2} G & 0
\end{array}\right) \bmod \Psi^{-2},
$$

thanks to (8) and (35)-(36).

As the two lines of the Calderón projector $C_{1}$ in (13) are the Cauchy data of a radiating electromagnetic field, according to the definition (25) $Y\left(\operatorname{Id} / 2-K^{\times}\right)=-z^{-1} T^{\times}$. And, because $K^{\times}$is well known to vanish on a flat surface, the admittance $Y$ on an infinite plane is

$$
Y=-2 z^{-1} T^{\times} .
$$

On the other hand, the complete symbol of $G$ on a an infinite plane being $\frac{1}{2 i}\left(k^{2}-\|\xi\|^{2}\right)^{-1 / 2}$, this operator appears as a fractional power of the Helmholtz equation:

$$
G=\frac{1}{2 i}\left(\Delta+k^{2} \mathrm{Id}\right)^{-1 / 2} .
$$

Combining (45), (46) and (47), we obtain on an infinite plane

$$
Y=\frac{1}{z k}\left(\begin{array}{cc}
0 & -\left(\Delta+k^{2} \mathrm{Id}\right)^{1 / 2} \\
k^{2}\left(\Delta+k^{2} \mathrm{Id}\right)^{-1 / 2} & 0
\end{array}\right) .
$$


So we have a representation of $Y$ on the plane through a formula easily generalizable to any kind of surface. Indeed, if $\Delta$ in (48) is viewed as the Laplace-Beltrami operator, this formula yields an approximation of the admittance for a general surface $\Gamma$.

But, taken as is, formula (48) would result in a GCSIE equation with spurious modes. A remedy is to localize it by replacing $k$ with $k+i \epsilon$, where $\epsilon>0$ is a small damping parameter. Finally, the approximation of $Y_{1}$ we propose is:

$$
\widetilde{Y}_{1}:=\frac{1}{z_{1} k_{1}}\left(\begin{array}{cc}
0 & -\left(\Delta+k_{1, \epsilon}^{2} \mathrm{Id}\right)^{1 / 2} \\
k_{1}^{2}\left(\Delta+k_{1, \epsilon}^{2} \mathrm{Id}\right)^{-1 / 2} & 0
\end{array}\right),
$$

where $k_{1, \epsilon}:=k_{1}+i \epsilon$ with $\epsilon>0$.

3.5. A well-posed equation. With an approximation of the optimal regularizing operator $R$ gkiven as $\widetilde{R}=\left(\widetilde{R}_{1}, \widetilde{R}_{1}-\mathrm{Id}\right)$, the GCSIE equation (24) becomes

$$
C_{1} \widetilde{R}_{1}+C_{2}\left(\operatorname{Id}-\widetilde{R}_{1}\right)(\mathbf{u}, \mathbf{v})=\left(\mathbf{u}_{0}, \mathbf{v}_{0}\right) .
$$

For the sequel,

$$
\widetilde{R}_{1}:=\left(\begin{array}{cc}
\widetilde{A} & -z_{1}^{2} \widetilde{Y}_{1} \widetilde{B} \\
\widetilde{Y}_{1} \widetilde{A} & \widetilde{B}
\end{array}\right),
$$

where $\widetilde{A}, \widetilde{B}$ and $\widetilde{Y}_{1}$ are given by (40), (41) and (49), respectively. The goal of this paragraph is to show that equation (50) is well-posed.

Proposition 3.6. If a tangential vector field $(\mathbf{u}, \mathbf{v})$ on $\Gamma$ verifies $\widetilde{R}(\mathbf{u}, \mathbf{v})=(\mathbf{u}, \mathbf{v})$, then $\widetilde{Y}_{1} \mathbf{u}=\mathbf{v}$.

Proof. If $\widetilde{R}(\mathbf{u}, \mathbf{v})=(\mathbf{u}, \mathbf{v})$, then $\widetilde{Y}_{1} \widetilde{A} \mathbf{u}+\widetilde{B} \mathbf{v}=\mathbf{v}$, we can rewrite $\widetilde{Y}_{1}^{\prime} \mathbf{n} \times \widetilde{A} \mathbf{n} \times \mathbf{n} \times \mathbf{u}=(\operatorname{Id}-\widetilde{B}) \mathbf{v}$ with $\widetilde{Y}_{1}^{\prime}:=\widetilde{Y}_{1} \mathbf{n} \times$. As relation (42) is equivalent to $\mathbf{n} \times \widetilde{A} \mathbf{n} \times=(\widetilde{B}-\mathrm{Id})$, we have $\widetilde{Y}_{1}^{\prime}(\widetilde{B}-\mathrm{Id}) \mathbf{n} \times \mathbf{u}=(\mathrm{Id}-\widetilde{B}) \mathbf{v}$, which yields $\widetilde{Y}_{1} \mathbf{u}=\mathbf{v}$ because $(\widetilde{B}-\mathrm{Id})$ and $\widetilde{Y}_{1}^{\prime}$ commute.

If $H^{s}$ denotes the usual Sobolev space of vector fields on $\Gamma$ whose norm is written $|\cdot|_{s}$, we define $H^{s, s^{\prime}}$ as the functional space composed of any tangential field $\mathbf{u}$ such that $\prod_{\text {loop }} \mathbf{u} \in H^{s}$ and $\prod_{\text {star }} \mathbf{u} \in H^{s^{\prime}}$. 
Equipped with the norm $\left(\left|\prod_{\text {loop }} \mathbf{u}\right|_{s}^{2}+\left|\prod_{\text {star }} \mathbf{u}\right|_{s^{\prime}}^{2}\right)^{1 / 2}, H^{s, s^{\prime}}$ becomes a Hilbert space.

We are going to see that a relevant space for our equation is $X:=H^{0,1 / 2}$. It is worthwhile mentioning that $X$ can be defined in a less abstract manner. Indeed, if $H_{\mathrm{div}}^{-1 / 2}$ is the space of all tangential vectorial fields in $H^{-1 / 2}$ with divergence in $H^{-1 / 2}$, the space $X$ is nothing else than $L_{T}^{2} \cap H_{\text {div }}^{-1 / 2}$. For details, we refer the reader to [19] where $X$ is used to achieve the numerical analysis of the combined field integral equation of the electromagnetism.

A convenient way of expressing that $M_{i j} \in \Psi^{m_{i j}}$ for all $i, j=1,2$ in (43) is to write

$$
M \in \Psi\left(\begin{array}{ll}
m_{11} & m_{12} \\
m_{21} & m_{22}
\end{array}\right) .
$$

When all orders $m_{i j}$ are the same, say $m$, we will just write $\Psi^{m}$.

Proposition 3.7. It is known that $K^{\times} \in \Psi^{-1}$, but the matrix representation (43) shows that $K^{\times}$is a little bit more regular:

$$
K^{\times} \in \Psi\left(\begin{array}{ll}
-1 & -1 \\
-3 & -1
\end{array}\right) .
$$

Proof. Starting from the Calderón relation Id $=-4\left(T^{\times^{2}}-K^{\times^{2}}\right)$, one has modulo $\Psi^{-3}, \prod_{\text {star }} K^{\times} \prod_{\text {loop }}=-4 \prod_{\text {star }} K^{\times} T^{\times^{2}} \prod_{\text {loop }}$, which is equal to $4 \prod_{\text {star }} T^{\times} K^{\times} T^{\times} \prod_{\text {loop }}$, due to the anti-commuting relation (15). As $\prod_{\text {star }} T^{\times}$and $T^{\times} \prod_{\text {loop }}$ are in $\Psi^{-1}, K_{21}^{\times} \in \Psi^{-3}$ and (53) is shown.

If $\mathbf{u}, \mathbf{v}$ are some vector fields on $\Gamma$, we denote by $\langle\mathbf{u}, \mathbf{v}\rangle:=\int \mathbf{u} \cdot \mathbf{n} \times$ $\overline{\mathbf{v}} d \Gamma$. Related to this duality product is a classical result, a consequence of the famous Rellich's lemma (see, for instance, [13, Theorem 6.10]):

Proposition 3.8. Any radiating electromagnetic field $(\mathbf{E}, \mathbf{H})$ on $\mathbb{R}^{3} \backslash \Gamma$, non-null on $\Omega_{1}$, satisfies

$$
\begin{aligned}
& \Re\left\langle\gamma_{1} \mathbf{H}, \gamma_{1} \mathbf{E}\right\rangle>0 \\
& \Re\left\langle\gamma_{2} \mathbf{H}, \gamma_{2} \mathbf{E}\right\rangle=0 .
\end{aligned}
$$


Proposition 3.9. If $\mathbf{u}$ is a non-null tangential vector field on $\Gamma$, then $\Re\left\langle\widetilde{Y}_{1} \mathbf{u}, \mathbf{u}\right\rangle$ and $\Re\left\langle\widetilde{Y}_{1} \widetilde{B} \mathbf{u}, \mathbf{u}\right\rangle$ are both $>0$.

Proof. Let $p:=P_{\text {loop }} \mathbf{u}$ and $q:=P_{\text {star }} \mathbf{u}$ be the Helmholtz potentials of $\mathbf{u}$. Then

$$
\begin{aligned}
& z_{1} k_{1} \Re\left\langle\widetilde{Y}_{1} \mathbf{u}, \mathbf{u}\right\rangle=k_{1}^{2} \Re\left\langle\left(\Delta+k_{1, \varepsilon}^{2} \mathrm{Id}\right)^{-1 / 2} p, p\right\rangle \\
& \Re\left\langle\left(\Delta+k_{1, \varepsilon}^{2} \mathrm{Id}\right)^{1 / 2} q, q\right\rangle \text {. }
\end{aligned}
$$

As the spectrum of $\Delta$ lies on the negative real axis, the spectral theorem states that the real part of the spectrum of $\left(\Delta+k_{1, \varepsilon}^{2} \mathrm{Id}\right)^{ \pm 1 / 2}$ is strictly positive. Therefore, $\Re\left\langle\widetilde{Y}_{1} \mathbf{u}, \mathbf{u}\right\rangle>0$ and, with the same reasoning, $\Re\left\langle\widetilde{Y}_{1} \widetilde{B} \mathbf{v}, \mathbf{v}\right\rangle>0$.

Theorem 3.10. The operator $C_{1} \widetilde{R}_{1}+C_{2}\left(\mathrm{Id}-\widetilde{R}_{1}\right)$ is a one-to-one (bijective) mapping on $X$.

Proof. We will apply the Fredholm alternative to $Z:=C_{1} \widetilde{R}_{1}+$ $C_{2}\left(\mathrm{Id}-\widetilde{R}_{1}\right)$ seen as an injective compact perturbation of identity on $X$. More exactly, we will show that $Z$ is equal to the identity plus an operator belonging to a special class of pseudo-differential operators

$$
\Psi^{c}:=\Psi\left(\begin{array}{cc}
-1 & 0 \\
-2 & -1
\end{array}\right),
$$

all of whose elements are compact in $X$ because of the mapping $H^{0,1 / 2}$ to $H^{1 / 2,3 / 2}$.

Let us first establish some technical results:

$$
\begin{aligned}
2\left(z_{1} T_{1}^{\times}+z_{2} T_{2}^{\times}\right) \widetilde{Y}_{1} \widetilde{A} & =\operatorname{Id} \bmod \Psi^{c} \\
2 z_{1} z_{2}^{-1}\left(z_{2} T_{1}^{\times}+z_{1} T_{2}^{\times}\right) \widetilde{Y}_{1} \widetilde{B} & =\operatorname{Id} \bmod \Psi^{c} \\
z_{1}^{-1} T_{1}^{\times} \widetilde{A}-z_{2}^{-1} T_{2}^{\times}(\operatorname{Id}-\widetilde{A}) & =0 \bmod \Psi^{c} \\
z_{1} T_{1}^{\times} \widetilde{B}-z_{2} T_{2}^{\times}(\operatorname{Id}-\widetilde{B}) & =0 \bmod \Psi^{c} \\
K^{\times} \widetilde{Y}_{1} \widetilde{A} & =0 \bmod \Psi^{c} \\
K^{\times} \widetilde{Y}_{1} \widetilde{B} & =0 \bmod \Psi^{c} .
\end{aligned}
$$


The identities (57) and (58) are trivial consequences of definitions of $\widetilde{A}$ and $\widetilde{B}$ at the condition to verify that

$$
2 z_{1} T_{j}^{\times} \widetilde{Y}_{1}=\frac{k_{1}}{k_{j}} \prod_{\text {loop }}+\frac{k_{j}}{k_{1}} \prod_{\text {star }} \bmod \Psi^{c} .
$$

As from (49),

$$
\tilde{Y}_{1} \in \Psi\left(\begin{array}{cc}
-2 & 1 \\
-1 & -2
\end{array}\right)
$$

the product of an operator of class $\Psi^{-2}$ with $\widetilde{Y}_{1}$ is in $\Psi^{c}$. Hence, combining (45) and (49) shows that, modulo $\Psi^{c}, z_{1} T_{j}^{\times} \widetilde{Y}_{1}$ is equal to

$$
-\frac{1}{i k_{j} k_{1}}\left(\begin{array}{cc}
G_{j}\left(\Delta+k_{j}^{2} \mathrm{Id}\right) k_{1}^{2}\left(\Delta+k_{1, \varepsilon}^{2} \mathrm{Id}\right)^{1 / 2} & 0 \\
0 & k_{j}^{2} G_{j}\left(\Delta+k_{1, \varepsilon}^{2} \mathrm{Id}\right)^{1 / 2}
\end{array}\right) .
$$

We then derive (63) by a simple pseudo-differential calculus involving the principal symbols of $G_{j},\left(\Delta+k_{j}^{2} \mathrm{Id}\right)$ and $\left(\Delta+k_{1, \varepsilon}^{2} \mathrm{Id}\right)^{ \pm 1 / 2}$ given, respectively, by $-\|\xi\|^{-1} / 2,-\|\xi\|^{2}$ and $(i\|\xi\|)^{ \pm 1}$.

Expanding $T_{1}^{\times}, T_{2}^{\times}$and $B$ with (45), (41) shows that, modulo $\Psi^{c}$, $z_{1} T_{1}^{\times} \widetilde{B}-z_{2} T_{2}^{\times}(\mathrm{Id}-\widetilde{B})$ is equal to

$$
\left(\begin{array}{cc}
0 & \beta\left(G_{1}\left(\Delta-k_{1}^{2} \mathrm{Id}\right)-G_{2}\left(\Delta-k_{2}^{2} \mathrm{Id}\right)\right) \\
\alpha\left(G_{2}-G_{1}\right) & 0
\end{array}\right)
$$

with $\alpha=i z_{1} z_{2} k_{1} k_{2}\left(z_{1} k_{1}+z_{2} k_{2}\right)^{-1}, \beta=i z_{1} z_{2}\left(z_{2} k_{1}+z_{1} k_{2}\right)^{-1}$. This results in (60) because $G_{1}$ and $G_{2}$ are operators of class $\Psi^{-1}$ with the same principal symbol. We could show this in the same manner as (59).

With (53) and (64), we have $K \times \widetilde{Y}_{1} \in \Psi\left(\begin{array}{cc}-2 & 0 \\ -2 & -2\end{array}\right)$, leading to (61) and (62).

Using (13) and (51), each coefficient-operator $Z_{i j}$ of $C_{1} \widetilde{R}_{1}+C_{2}(\mathrm{Id}-$ $\left.\widetilde{R}_{1}\right)$ can be read as:

$$
\begin{aligned}
Z_{11}= & \left(\frac{\operatorname{Id}}{2}-K_{1}^{\times}\right) \widetilde{A}+z_{1} T_{1}^{\times} \widetilde{Y}_{1} \widetilde{A} \\
& +\left(\frac{\operatorname{Id}}{2}+K_{2}^{\times}\right)(\operatorname{Id}-\widetilde{A})+z_{2} T_{2}^{\times} \widetilde{Y}_{1} \widetilde{A}
\end{aligned}
$$




$$
\begin{aligned}
Z_{12}= & -\left(\frac{\mathrm{Id}}{2}-K_{1}^{\times}\right) z_{1}^{2} \widetilde{Y}_{1} \widetilde{B} \\
& +z_{1} T_{1}^{\times} \widetilde{B}+\left(\frac{\mathrm{Id}}{2}+K_{2}^{\times}\right) z_{1}^{2} \widetilde{Y}_{1} \widetilde{B} \\
& -z_{2} T_{2}^{\times}(\mathrm{Id}-\widetilde{B}) \\
Z_{21}= & -z_{1}^{-1} T_{1}^{\times} \widetilde{A}+\left(\frac{\mathrm{Id}}{2}-K_{1}^{\times}\right) \widetilde{Y}_{1} \widetilde{A} \\
& +z_{2}^{-1} T_{2}^{\times}(\mathrm{Id}-\widetilde{A})-\left(\frac{\mathrm{Id}}{2}+K_{2}^{\times}\right) \widetilde{Y}_{1} \widetilde{A} \\
Z_{22}= & z_{1}^{-1} T_{1}^{\times} z_{1}^{2} \widetilde{Y}_{1} \widetilde{B}+\left(\frac{\operatorname{Id}}{2}+K_{1}^{\times}\right) \widetilde{B} \\
& +z_{2}^{-1} T_{2}^{\times} z_{1}^{2} \widetilde{Y}_{1} \widetilde{B}+\left(\frac{\operatorname{Id}}{2}+K_{2}^{\times}\right)(\operatorname{Id}-\widetilde{B}) .
\end{aligned}
$$

Therefore, modulo $\Psi^{c}$, we have $Z_{11}=$ Id because of (53) and (57), $Z_{12}=0$ because of $(60)$ and (62), $Z_{21}=0$ because of (59) and (61), $Z_{22}=$ Id because of (53) and (58), proving that $Z$ is a compact perturbation of the identity on $X$. Now, let us show the injectivity.

Let $(\mathbf{u}, \mathbf{v}) \in X$ be such that $Z(\mathbf{u}, \mathbf{v})=0$. As the transmission problem (1)-(3) is well posed, the two following electromagnetic fields

$$
\left(\mathbf{E}_{1}, \mathbf{H}_{1}\right):=\mathbf{C}_{1} \widetilde{R}_{1}(\mathbf{u}, \mathbf{v}), \quad\left(\mathbf{E}_{2}, \mathbf{H}_{2}\right):=\mathbf{C}_{2}\left(\operatorname{Id}-\widetilde{R}_{1}\right)(\mathbf{u}, \mathbf{v})
$$

are null, respectively, on $\Omega_{1}$ and $\Omega_{2}$. From (13), we have the jump relations

$$
\left(\gamma_{1}-\gamma_{2}\right) \mathbf{C} 1=\mathrm{Id}, \quad\left(\gamma_{2}-\gamma_{1}\right) \mathbf{C} 2=\mathrm{Id},
$$

which applied to (65) lead to

$$
\begin{aligned}
& -\gamma_{2} \mathbf{E}_{1}=\widetilde{R}_{11}^{1} \mathbf{u}+\widetilde{R}_{12}^{1} \mathbf{v}, \quad \gamma_{1} \mathbf{E}_{2}=\mathbf{u}-\widetilde{R}_{11}^{1} \mathbf{u}-\widetilde{R}_{12}^{1} \mathbf{v} \\
& -\gamma_{2} \mathbf{H}_{1}=\widetilde{R}_{21}^{1} \mathbf{u}+\widetilde{R}_{22}^{1} \mathbf{v}, \quad-\gamma_{1} \mathbf{H}_{2}=\mathbf{v}-\widetilde{R}_{21}^{1} \mathbf{u}-\widetilde{R}_{22}^{1} \mathbf{v} \text {. }
\end{aligned}
$$

We leave it to the reader to verify that $\mathbf{n} \times \widetilde{Y}_{1}$ and $\mathbf{n} \times \widetilde{R}_{i j}^{1}$ go from $X=H^{0,1 / 2}$ to its dual $X^{*}=H^{0,-1 / 2}$, allowing to compute, with the help of (66), the duality $\left\langle\gamma_{1} \mathbf{H}_{2}, \gamma_{1} \mathbf{E}_{2}\right\rangle=\left\langle\gamma_{2} \mathbf{H}_{1}, \gamma_{2} \mathbf{E}_{1}\right\rangle+\langle\mathbf{v}, \mathbf{u}\rangle-$ $\left\langle\mathbf{v}, \widetilde{R}_{11}^{1} \mathbf{u}\right\rangle-\left\langle\mathbf{v}, \widetilde{R}_{12}^{1} \mathbf{v}\right\rangle-\left\langle\widetilde{R}_{21}^{1} \mathbf{u}, \mathbf{u}\right\rangle-\left\langle\widetilde{R}_{22}^{1} \mathbf{v}, \mathbf{u}\right\rangle$. Relation (42) shows that $\langle\mathbf{v}, \widetilde{A} \mathbf{u}\rangle+\langle\widetilde{B} \mathbf{v}, \mathbf{u}\rangle=\langle\mathbf{v}, \mathbf{u}\rangle$, and so $\Re\left\langle\gamma_{1} \mathbf{H}_{2}, \gamma_{1} \mathbf{E}_{2}\right\rangle=-z_{1}^{2} \Re\left\langle\widetilde{Y}_{1} \widetilde{B} \mathbf{v}, \mathbf{v}\right\rangle-$ $\Re\left\langle\widetilde{Y}_{1} \mathbf{u}, \mathbf{u}\right\rangle$. We conclude with Proposition 9 that $\Re\left\langle\gamma_{1} \mathbf{H}_{2}, \gamma_{1} \mathbf{E}_{2}\right\rangle \leq 0$, 
which implies that $\mathbf{E}_{2}=\mathbf{H}_{2}=0$ on the whole space as a consequence of (54). Coming back to the right relations of (66) gives $\widetilde{R}_{1}(\mathbf{u}, \mathbf{v})=(\mathbf{u}, \mathbf{v})$; hence, $\widetilde{Y}_{1} \mathbf{u}=\mathbf{v}$ according to Proposition 6 . The left relations of (66) imply that $\Re\left\langle\gamma_{1} \mathbf{H} 2, \gamma_{1} \mathbf{E}_{2}\right\rangle=\Re\langle\mathbf{u}, \mathbf{v}\rangle=\Re\left\langle\mathbf{u}, \widetilde{Y}_{1} \mathbf{u}\right\rangle$ and, as $\left(\mathbf{E}_{2}, \mathbf{H}_{2}\right)$ is null, $\Re\left\langle\mathbf{u}, \widetilde{Y}_{1} \mathbf{u}\right\rangle=0$. Proposition 9 gives $\mathbf{u}=0$, and it follows that $\mathbf{v}=\widetilde{Y}_{1} \mathbf{u}=0$.

Finally, since $Z$ is an injective compact perturbation of the identity on $X$, it is an invertible operator according to the Fredholm alternative theorem.

4. Numerical issues. The aim of this part is not to present in details the discretization scheme of the GCSIE, which will be the subject of a future communication in order to give some preliminary results indicating good iterative behavior of the GCSIE (50) compared to the popular PMCHWT equation (18). If boundary operators are classically discretized following a Galerkin method on Raviart-Thomas elements, it is worthwhile to say that the synthesis of $\widetilde{Y}_{1}$ given by (49) is not straightforward. Indeed, taken as it is, this formula is numerically intractable because of the square roots of operators. A solution is to approach the functions $z^{1 / 2}$ and $z^{-1 / 2}$ in the vicinity of the spectrum of $\Delta+k_{1}^{2}$ with a sum of poles $\left(z-p_{i}\right)^{-1}$ given by a Padé expansion. We refer to [23] where the technique is presented in the context a GCSIE for Leontovich problems.

In the experiments that follow, both GCSIE and PMCHWT matrixvector products are accelerated with a multilevel fast multipole algorithm and iterative solutions are done with a regular GMRES solver whose stopping criterion on the residue is fixed to $10^{-3}$. For the PMCHWT equation an SPAI (SParse Approximate Inverse) preconditioner is used.

4.1. Analytical case: Dielectric sphere. We consider the transmission problem given by a plane wave impinging at $2 \mathrm{GHz}$ a dielectric unit sphere with constants $\varepsilon=4$ and $\mu=1$. The diameter of the sphere is about 26 wavelengths of a plane wave travelling in the dielectric.

The far field of the scattered field, named the bistatic radar cross section (RCS), is a good criterion for evaluating the accuracy of a solution. The RCS of a sphere can be analytically computed with the 
Mie series. In Figure 1 we observe a very good agreement between this reference solution and the results of the GCSIE and PMCHWT.

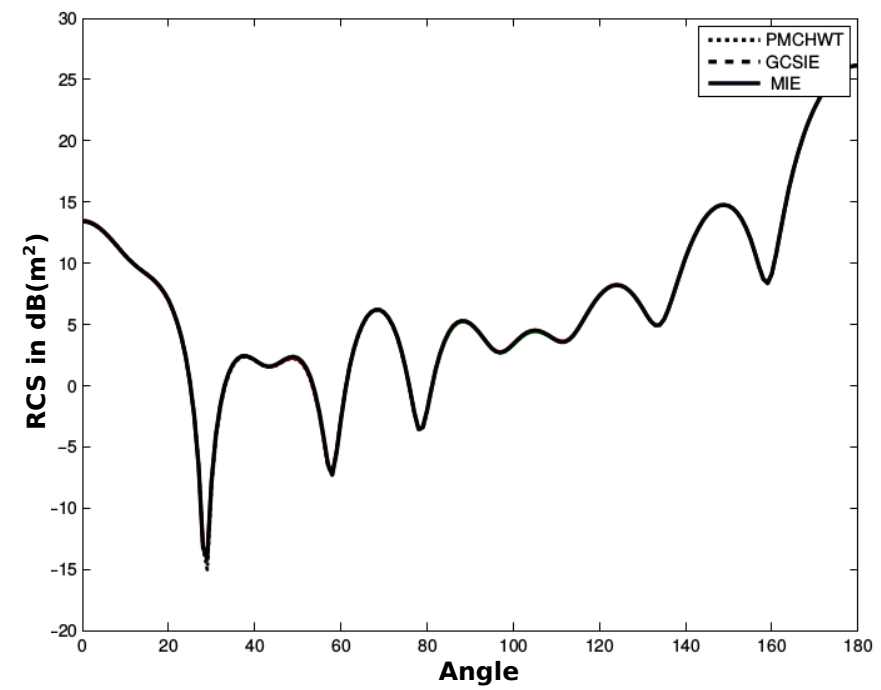

FiguRE 1. Bistatic radar cross section of the sphere at $2 \mathrm{GHz}$.

Figure 2 represents the spectrums of the GCSIE and the PMCHWT operators whose eigenvectors on a sphere are the spherical harmonics [15]. As expected, the spectrum of the GCSIE is bounded with an important accumulation of eigenvalues near $(1,0)$, meaning that $\widetilde{R}$ is a rather good approximation of the optimal regularizing operator $R$. By contrast, the spectrum of the PMCHWT turns around the origin $(0,0)$ and is spread more in the complex plane. This kind of distribution is less adapted to a fast iterative resolution.

As the convergence speed of an iterative solver also depends on the mesh, two homogeneous meshes of different sizes are tested. If $\lambda$ is the wave length of a plane wave travelling inside the sphere and $h$ is the average edge length of the mesh, then the ratio $\lambda / h$ measures the accuracy of the mesh. Table 1 shows that the number of matrix-vector products for the GCSIE is very stable with regard to the discretization of the geometry. This stability is not verified by the PMCHWT whose 


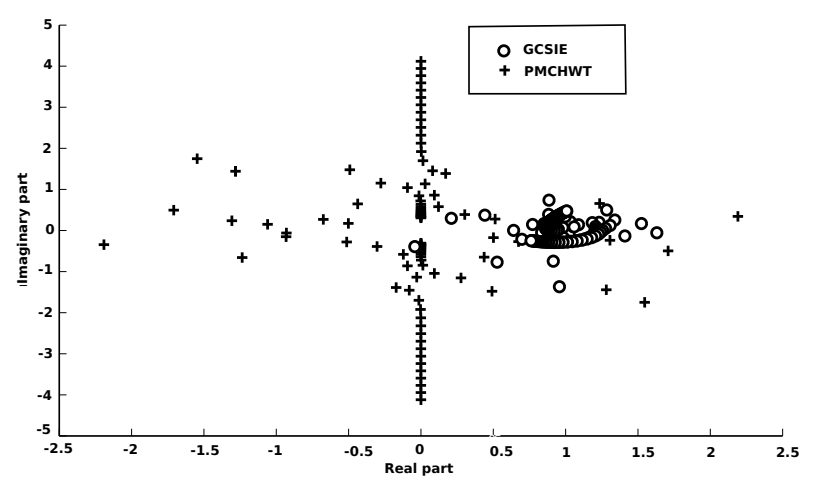

FIGURE 2. Spectrums of the GCSIE and PMCHWT (sphere at $225 \mathrm{MHz}$ ).

TABLE 1. Iterations count vs. mesh precision (sphere at $2 \mathrm{GHz}$ ).

\begin{tabular}{|l|l|l|}
\hline$\lambda / h$ & GCSIE & PMCHWT \\
\hline 7 & 23 it. & 40 it. \\
\hline 14 & 27 it. & 100 it. $\left(\right.$ res $\left.=10^{-2}\right)$ \\
\hline
\end{tabular}

TABLE 2. Iterations count vs. frequency at a fixed mesh precision $\lambda / h=14$ (sphere).

\begin{tabular}{|l|l|l|}
\hline frequency & GCSIE & PMCHWT \\
\hline $225 \mathrm{Mhz}$ & 23 it. & 40 it. \\
\hline $450 \mathrm{MHz}$ & 60 it. & 115 it. \\
\hline $900 \mathrm{MHz}$ & 115 it. & 300 it. $\left(\operatorname{res}=2 \cdot 10^{-3}\right)$ \\
\hline
\end{tabular}

iterations' count increases significantly with the size of the mesh, despite the use of the SPAI preconditioner.

It is also well known that the convergence speed of an iterative method is slowed down by the rise of the frequency. Table 2 presents results at different frequencies but with a fixed mesh precision $\lambda / h=14$ and a GMRES residual equal to $10^{-3}$. As expected, the number of iterations increases in function of the frequency, but the new formulation is significantly more stable than the PMCHWT. 


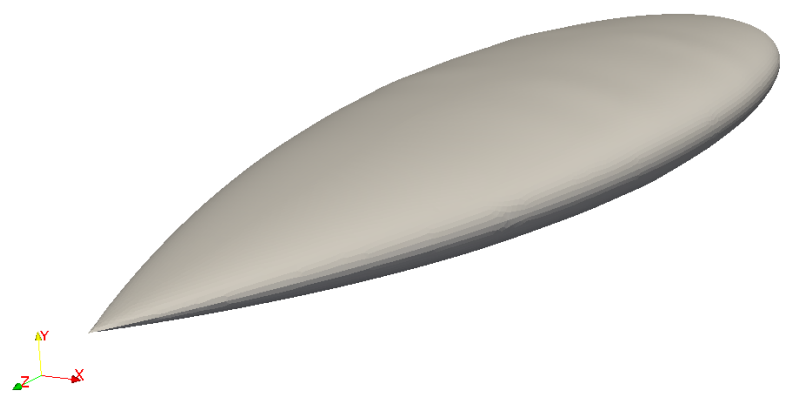

FiguRE 3. Geometry of the NASA almond.

4.2. Singular geometry: Dielectric almond. The NASA almond (Figure 3) is a popular benchmark in the community of computational electromagnetics. For our experiments, the almond is filled with a dielectric material of constants $\varepsilon=4$ and $\mu=1$ and has characteristic lengths about equal to $9 \lambda \times 3 \lambda \times 22 \lambda$ where $\lambda$ is the interior wavelength. The incident plane wave illuminates the almond by the tip at $1.3 \mathrm{GHz}$. The mesh is made of 134648 triangles and supports 201972 degrees of freedom corresponding to a precision $\lambda / h \sim 7$.

Figure 4 gives the bistatic RCS of the almond. Curves are very close to each other.

Figure 5 shows the history of the GMRES convergence for the GCSIE and SPAI preconditioned PMCHWT formulations. We see that the GCSIE formulation gives the solution with a smaller number of iterations. We have about a factor 7 between the two methods.

5. Conclusions. In this paper, we have proposed and analyzed a new GCSIE formulation for the scattering by homogeneous dielectric 3-D arbitrary shaped objects. In particular, for smooth obstacles, we have proved that this formulation is well-posed at any frequency and can be viewed as a compact perturbation of the identity operator. This formulation has been implemented in the context of a GMRES solution coupled with a multilevel fast multipole method. Some numerical comparisons with the so-called PMCHWT formulation have shown the efficiency of the new formulation: solutions of the GCSIE are 


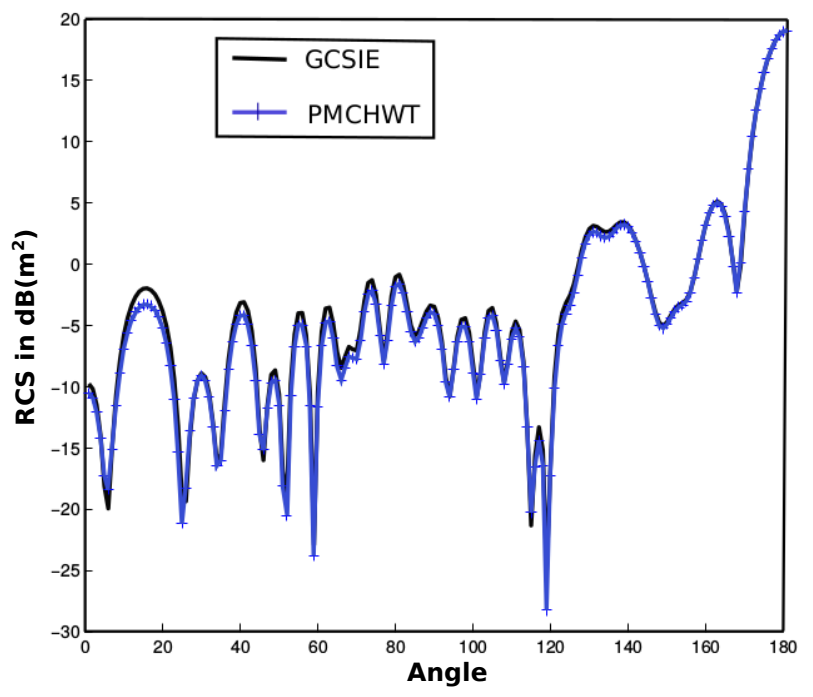

Figure 4. Bistatic Radar Cross section (almond at $1 \mathrm{GHz}$ ).

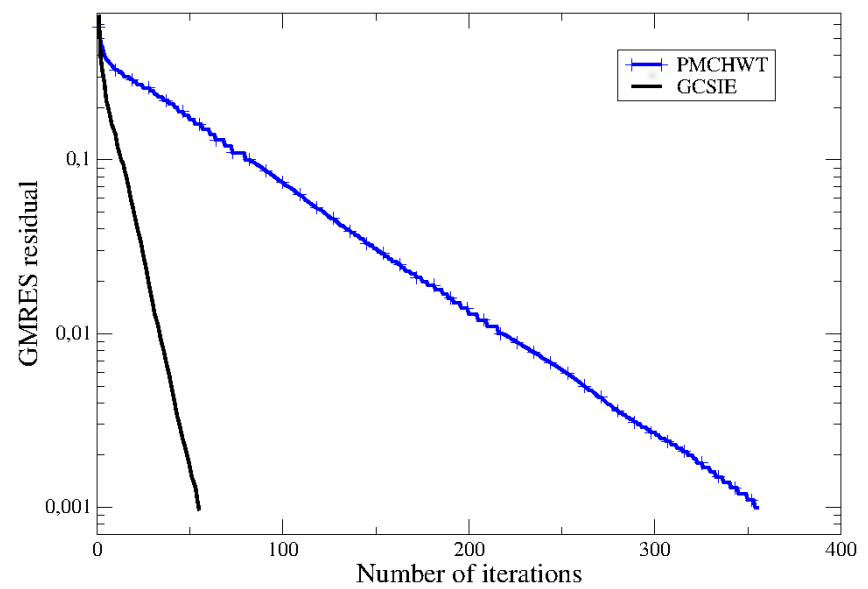

FIGURE 5. History of the GMRES convergence with a GCSIE and an SPAI preconditioned PMCHWT equation. 
obtained after a small number of iterations and with a similar accuracy as PMCHWT. In conclusion, this GCSIE formulation seems to be an interesting alternative to the classical formulations in the context of homogeneous dielectric obstacles.

\section{REFERENCES}

1. F.P. Andriulli, Well-posed boundary element formulations in electromagnetics, Ph.D. dissertation, University of Michigan.

2. F. Andriulli, K. Cools, H. Bagci, F. Olyslager, A. Buffa, S. Christiansen and E. Michielssen, A multiplicative Calderon preconditioner for the electric field integral equation, IEEE Trans. Ant. Prop. 56 (2008), 2398-2412.

3. S. Borel, D. Levadoux and F. Alouges, A new well-conditioned integral formulation for Maxwell equations in three-dimensions, IEEE Trans. Ant. Prop. 53 (2005), 2995-3004.

4. Y. Boubendir, O. Bruno, D. Levadoux and C. Turc, Integral equations requiring small numbers of Krylov-subspace iterations for two-dimensional smooth penetrable scattering problems, Appl. Numer. Math. 95 (2015), 82-98.

5. _ Regularized combined field integral equations for acoustic transmission problems, SIAM J. Appl. Math 75 (2015), 929-952.

6. O.P. Bruno, Computational electromagnetics and acoustics: Highorder solvers, highfrequency configurations, high-order surface representations, Oberwolfach Reports 5 (2007), 284-287.

7. O. Bruno, T. Elling, R. Paffenroth and C. Turc, Electromagnetic integral equations requiring small numbers of Krylov-subspace iterations, J. Comp. Phys. 228 (2009), 6169-6183.

8. A. Buffa, R. Hiptmair, T. von Petersdorff and C. Schwab, Boundary element methods for Maxwell transmission problems in Lipschitz domains, Numer. Math. 95 (2003), 459-485.

9. S.L. Campbell, I.C.F. Ipsen, C.T. Kelley and C.D. Meyer, GMRES and the minimal polynomial, BIT Numer. Math. 36, Springer, Netherlands, 1996.

10. S.L. Campbell, I.C.F. Ipsen, C.T. Kelley, C.D. Meyer and Z.Q. Xue, Convergence estimates for solution of integral equations with GMRES, J. Integral Equations Applications 8 (1996), 19-34.

11. B. Carpentieri, I.S. Duff and L. Giraud, Sparse pattern selection strategies for robust frobenius-norm minimization preconditioners in electromagnetism, Numer. Lin. Alg. Appl. 7 (2000), 667-685.

12. D. Colton and P. Kress, Integral equation methods in scattering, Wiley \& Sons, New York, 1983.

13. , Inverse acoustic and electromagnetic scattering theory, SpringerVerlag, New York, 1992.

14. H. Contopanagos, D. Dembart, M. Epton, J. Ottusch, V. Rokhlin, J. Visher and S. Vandzura, Well-conditioned boundary integral equations for three- 
dimensional electromagnetic scattering, IEEE Trans. Ant. Prop. 50 (2002), 18241830 .

15. M. Darbas, Generalized CFIE for the iterative solution of 3-D Maxwell equations, Appl. Math. Lett. 19 (2006), 834-839.

16. B. Jung, T. Sarkar and Y. Chung, A survey of various frequency domain integral equations for the analysis of scattering from three-dimensional dielectric objects, PIER 36 (2002), 193-246.

17. D. Levadoux, Etude d'une équation intégrale adaptée à la résolution haute fréquence de l'équation de helmholtz, Ph.D. thesis, Paris, 2001.

18. A new integral formalism for transmission problems of electromagnetism, 8th Inter. Conf. Math. Numer. Aspects Waves, Reading, UK, July 23-27, 2007, 90-92.

19. Some preconditioners for the CFIE equation of electromagnetism, Math. Meth. Appl. Sci. 31 (2008), 2015-2028.

20. D. Levadoux and B.L. Michielsen, Analysis of a boundary integral equation for high frequency helmholtz problems, in Proc. 4th Inter. Conf. Math. Numer. Aspects Wave Propag., Golden, Colorado, 1998, 765-767.

21. , Nouvelles formuations intégrales pour les problèmes de diffraction d'ondes, Math. Model. Numer. Anal. 38 (2004), 157-175.

22. D. Levadoux, F. Millot and S. Pernet, New trends in the preconditioning of integral equations of electromagnetism, in Mathematics in industry-scientific computing in electical engineering, J. Roos and Luis R.J. Costa, ed., Springer, New York, 2010.

23. An unpreconditioned boundaryintegral for iterative solution of scattering problems with non-constant Leontovitch impedance boundary conditions, Comm. Comp. Phys. 15 (2014), 1431-1460.

24. J.C. Nédélec, Acoustic and electromagnetic equations: Integral representation for harmonic problems, Springer-Verlag, New York, 2001.

25. S. Pernet, A well-conditioned integral equation for iterative solution of scattering problems with a variable Leontovitch boundary condition, Math. Model. Numer. Anal. 44 (2010), 781-801.

26. E.P. Stephan, Boundary Integral equation for mixed boundary value problems, screen and transmission problems in $R^{3}$, Habilitationsschrift, Tech. Hochschule, Darmstadt, 1984.

Onera, Chemin de la Hunière et des Joncherettes,BP 80100, FR-91123, Palaiseau CEDEX

Email address: david.levadoux@onera.fr

CERFACS, 42 avenue Gaspard Coriolis, 31057 Toulouse

Email address: millot@cerfacs.fr

ONERA, 2 avenue Edouard Belin, BP 74025, FR-31055 Toulouse Cedex 4 Email address: Sebastien.Pernet@onera.fr 